\title{
Zika Virus
}

Created by: Marciano Paes

Revised by: Kíssila Rabelo

Version received: 20 May 2020

check for

updates

Zika virus (ZIKV) is an emergent arthropod-borne virus whose outbreak in Brazil has brought major public health problems. Infected individuals have different symptoms, including rash and pruritus, which can be relieved by the administration of antiallergics. In the case of pregnant women, ZIKV can cross the placenta and infect the fetus leading to congenital defects. We have identified that mast cells in the placentae of patients who had Zika during pregnancy can be infected. This led to our investigation on the possible role of mast cells during a ZIKV infection, using the HMC-1 cell line. We analyzed their permissiveness to infection, release of mediators and ultrastructural changes. Flow cytometry detection of ZIKV-NS1 expression $24 \mathrm{~h}$ post infection in $45.3 \%$ of cells showed that HMC1 cells are permissive to ZIKV infection. Following infection, $\beta$-hexosaminidase was measured in the supernatant of the cells with a notable release at $30 \mathrm{~min}$. In addition, an increase in TNF- $\alpha$, IL-6, IL-10 and VEGF levels were measured at $6 \mathrm{~h}$ and $24 \mathrm{~h}$ post infection. Lastly, different intracellular changes were observed in an ultrastructural analysis of infected cells. Our findings suggest that mast cells may represent an important source of mediators that can activate other immune cell types during a ZIKV infection, which has the potential to being a major contributor in the spread of the virus in cases of vertical transmission.
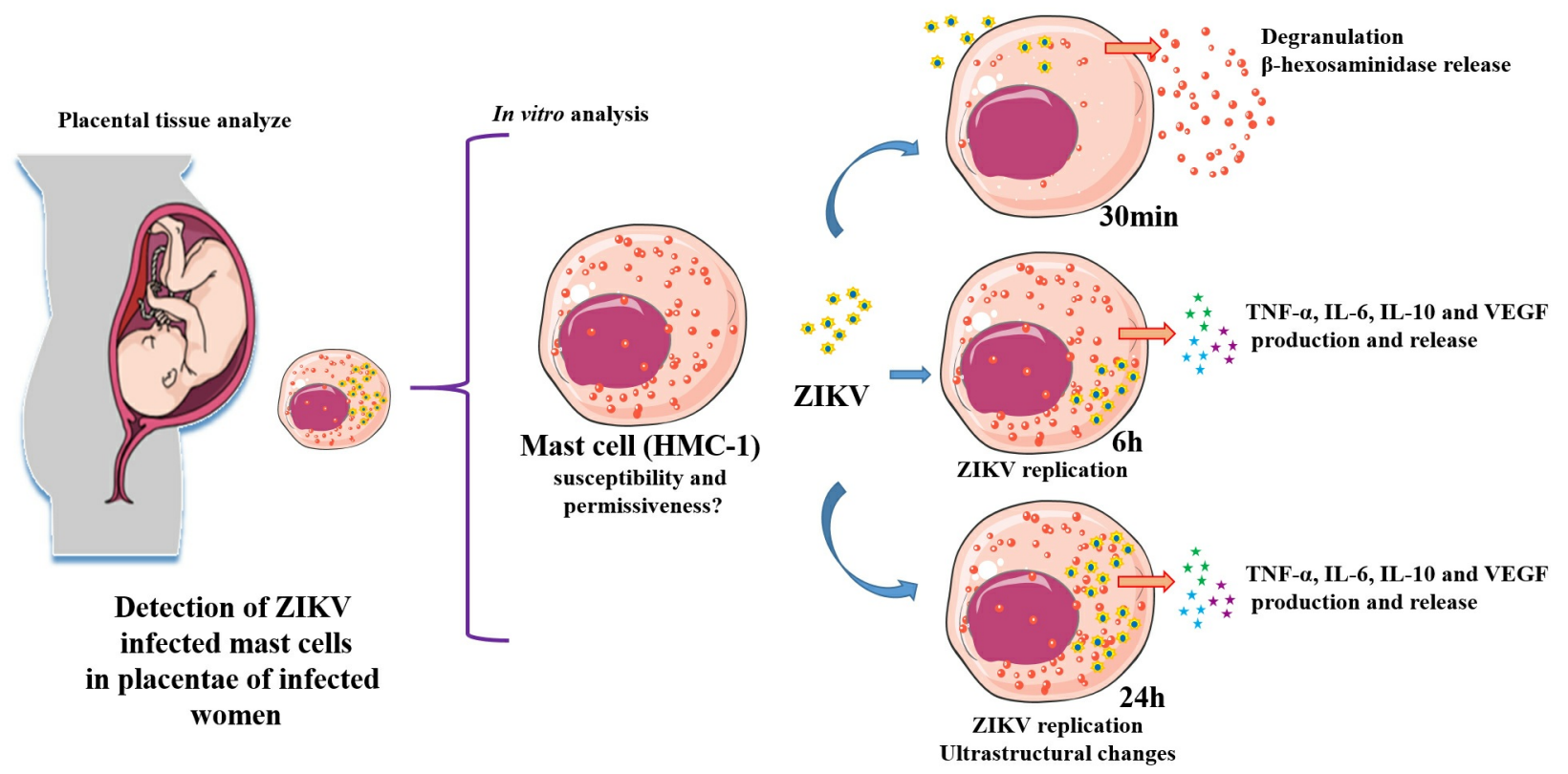

Zika fever is an important Arbovirus-caused disease that has surfaced in numerous countries in Asia, Africa and America [1]. The etiological agent of this disease, Zika virus (ZIKV), was initially isolated in 1947 from the blood of sentinel Rhesus monkeys during a study on yellow fever transmission in the Zika forest of Uganda, which gave rise to its name [2][3]. Transmission of the ZIKV is primarily through bites of infectedAedes mosquitos, with the most common vectors being Aedes aegypti and Aedes albopictus, but it can also happen by vertical transmission[4]. As a result of vertical transmission, there were alarming cases of Congenital Zika Syndrome, as the virus could cause damage to the placenta, infect placental cells and reach the fetus [5]. A ZIKV particle has a diameter of 25-30 $\mathrm{nm}$ and is a member of the Flaviviridae family that shares many similarities with other more widely known related viruses such as dengue, West Nile, Japanese encephalitis and yellow fever [6]. It has a single-stranded RNA genome with a positive polarity of $11 \mathrm{~Kb}$ and encodes a polyprotein precursor that is processed into the structural proteins such as capsid (C), pre-membrane (prM) and envelope (E) along with seven non-structural proteins (NS1, NS2A, NS2B, NS3, NS4A, NS4B and NS5) [7][8]. 
Mast cells are resident immunological cells found abundantly in tissues such as skin, endometrium and placenta that have prominent roles in immunologic reactions [9][10][11]. Their presence and prevalence in these tissues, along with their proximity to blood vessels, predispose these cells to be among the first immune cells that can be infected by ZIKV after a mosquito bite penetrates the skin. As some of the most frequent symptoms of zika are rash and pruritus, which are relieved by the administration of antiallergic drugs (anti-histamines), this has led us to believe that mast cells can play a role, although not yet elucidated, in the pathogenesis of the disease [12][13][14]. We hypothesize that it may be one of the cells involved in placental infections, which can directly contribute to vertical transmission.

Although there are no studies in the literature that have investigated the involvement of mast cells in a ZIKV infection to date, mast cells have a proven role in infections by dengue, another Arbovirus. Several products originating from mast cells are found at high levels in patients infected by dengue, especially those with plasma leakage [15][16]. While mast cells are permissive to dengue infection, it is most probable that they display a low level of the specific receptors required since the quantity of virus necessary to successfully infect this cell type is always higher than is needed for macrophages and dendritic cells [15][17][18].

HMC-1 cells are a lineage of human mast cells that characteristically express the cytokine receptor c-Kit abundantly and release different cytokines after degranulation stimuli. This cell line possesses the features necessary to serve as an in vitro model for the development of studies on mast cells ${ }^{[19]}$. HMC-1 has been widely used in studies on degranulation studies, endothelial activation and its interaction with other arboviruses [20][21].

\section{References}

1. Didier Musso; Albert I. Ko; David Baud; Zika Virus Infection - After the Pandemic.. New England Journal of Medicine 2019, 381, 1444-1457, 10.1056/NEJMra1808246.

2. Didier Musso; Duane J. Gubler; Zika Virus. Clinical Microbiology Reviews 2016, 29, 487-524, 10.1128/CMR.00072-15.

3. A.W.R. McCrae; B.G. Kirya; Yellow fever and Zika virus epizootics and enzootics in Uganda. Transactions of The Royal Society of Tropical Medicine and Hygiene 1981, 76, 552-562, 10.1016/0035-9203(82)90161-4.

4. Helen M. LaZear; Michael S. Diamond; Zika Virus: New Clinical Syndromes and Its Emergence in the Western Hemisphere. Journal of Virology 2016, 90, 4864-4875, 10.1128/JVI.00252-16.

5. Franciane Mouradian Emidio Teixeira; Anna Julia Pietrobon; Luana De Mendonça Oliveira; Luanda Mara Da Silva Oliveira; Maria Notomi Sato; Maternal-Fetal Interplay in Zika Virus Infection and Adverse Perinatal Outcomes.. Frontiers in Immunology 2020, 11, 175, 10.3389/fimmu.2020.00175.

6. O. Faye; C.C.D.M. Freire; J.V. De Oliveira; P.M.D.A. Zanotto; M. Diallo; A. A. Sall; Molecular evolution of Zika virus during its emergence in the 20th century. International Journal of Infectious Diseases 2014, 21, 2-3, 10.1016/j.ijid.2014.03.411.

7. Abdelrahman Ibrahim Abushouk; Ahmed Negida; Hussien Ahmed; An updated review of Zika virus. Journal of Clinical Virology 2016, 84, 53-58, 10.1016/j.jcv.2016.09.012.

8. Thomas J. Chambers; Chang S. Hahn; Ricardo Galler; Charles M. Rice; Flavivirus Genome Organization, Expression, and Replication. Annual Review of Microbiology 1990, 44, 649-688, 10.1146/annurev.mi.44.100190.003245.

9. R.A.J. Eady; T. Cowen; T.F. Marshall; Valerie Plummer; M.W. Greaves; Mast cell population density, blood vessel density and histamine content in normal human skin. British Journal of Dermatology 1979, 100, 623-633, 10.1111/j.1365-2133.1979.tb08065.x.

10. Magda Babina; Sven Guhl; Metin Artuc; Neil N. Trivedi; Torsten Zuberbier; Phenotypic variability in human skin mast cells. Experimental Dermatology 2016, 25, 434-439, 10.1111/exd.12924.

11. Youssef Derbala; Haidy Elazzamy; Mahmood Bilal; Rachel Reed; Maria Dinorah Salazar Garcia; Annie Skariah; Svetlana Dambaeva; Emilio Fernandez; Alfredo Germain; Alice Gilman-Sachs; et al.Kenneth BeamanJoanne Kwak-Kim Mast cell-induced immunopathology in recurrent pregnancy losses.. American Journal of Reproductive Immunology 2019, 82, e13128, 10.1111/aji.13128.

12. Humberto Guanche Garcell; Francisco Gutiérrez García; Manuel Ramirez Nodal; Alfredo Ruiz Lozano; Carlos R. Pérez Díaz; Anaika González Valdés; Luis Gonzalez Alvarez; Clinical relevance of Zika symptoms in the context of a Zika Dengue epidemic. Journal of Infection and Public Health 2020, 13, 173-176, 10.1016/j.jiph.2019.07.006.

13. Allison A. Harris; History, Epidemiology, and Clinical Manifestations of Zika: A Systematic Review. The Journal of Emergency Medicine 2016, 51, 216, 10.1016/j.jemermed.2016.06.035.

14. Ashok Munjal; Rekha Khandia; Kuldeep Dhama; Swati Sachan; Kumaragurubaran Karthik; Ruchi Tiwari; Yashpal S. Malik; Deepak Kumar; Raj K. Singh; Hafiz M.N. Iqbal; et al.Sunil K. Joshi Advances in Developing Therapies to Combat Zika Virus: Current Knowledge and Future Perspectives. Frontiers in Microbiology 2017, 8, 1469, 10.3389/fmicb.2017.01469.

15. Ayesa Syenina; Cyril J Jagaraj; Siti Ab Aman; Aishwarya Sridharan; Ashley L. St. John; Dengue vascular leakage is augmented by mast cell degranulation mediated by immunoglobulin Fcy receptors. eLife 2015, 4, 1-10, 10.7554/eLife.05291. 
16. Takahisa Furuta; Lyre Anni Murao; Nguyen Thi Phuong Lan; Nguyen Tien Huy; Lan Nguyen Thi Phuong; Tran Thi Thuy; Vo Dinh Tham; Cao Thi Phi Nga; Tran Thi Ngoc Ha; Yasukazu Ohmoto; et al.Mihoko KikuchiKouichi MoritaMichio YasunamiKenji HirayamaNaohiro Watanabe Association of Mast Cell-Derived VEGF and Proteases in Dengue Shock Syndrome. PLOS Neglected Tropical Diseases 2012, 6, 1-8, 10.1371/journal.pntd.0001505.

17. Andrea Troupin; Devon Shirley; Berlin Londono; Alan Watson; Cody McHale; Alex Hall; Adam Hartstone-Rose; William B. Klimstra; Gregorio Gomez; Tonya Colpitts; et al. A Role for Human Skin Mast Cells in Dengue Virus Infection and Systemic Spread. The Journal of Immunology 2016, 197, 4382-4391, 10.4049/jimmunol.1600846.

18. Berlin Londono; Julio C. Marinez-Angarita; Andrea Troupin; Tonya Colpitts; Role of Mast Cells in Dengue Virus Pathogenesis. DNA and Cell Biology 2017, 36, 423-427, 10.1089/dna.2017.3765.

19. G Nilsson; T Blom; M Kusche-Gullberg; L Kjellén; J H Butterfield; C Sundström; K Nilsson; L Hellman; Phenotypic characterization of the human mast-cell line HMC-1.. Scandinavian Journal of Immunology 1994, 39, 1-5.

20. David E. Cochrane; Robert E. Carraway; Kimberly Harrington; Melissa Laudano; Stephen A. Rawlings; Ross S. Feldberg; HMC-1 human mast cells synthesize neurotensin (NT) precursor, secrete bioactive NT-like peptide(s) and express NT receptor NTS1. Inflammation Research 2011, 60, 1139-1151, 10.1007/s00011-011-0378-6.

21. Mark Gilchrist; A. Dean Befus; Interferon-? regulates chemokine expression and release in the human mast cell line HMC1: role of nitric oxide. Immunology 2007, 123, 209-217, 10.1111/j.1365-2567.2007.02688.x.

\section{Keywords}

Zika virus; mast cells; placenta 